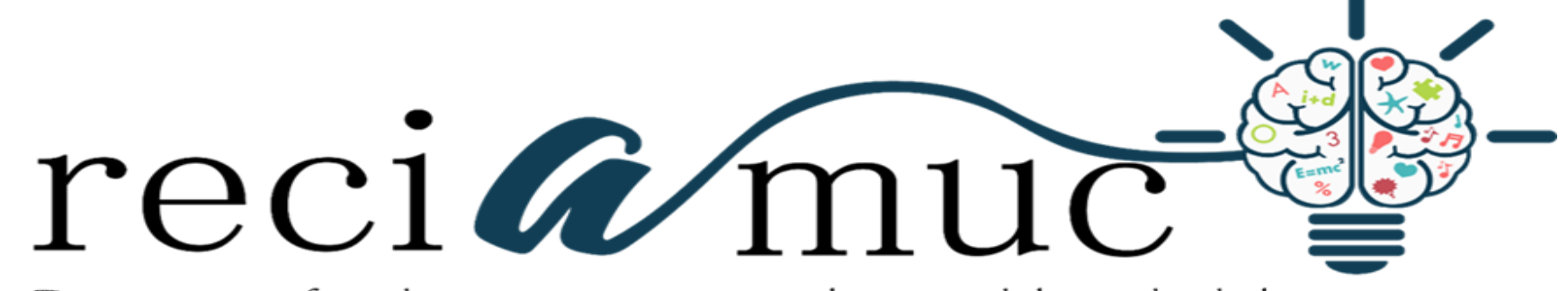

Revista cientifica de investigación actualización del mundo de las ciencias

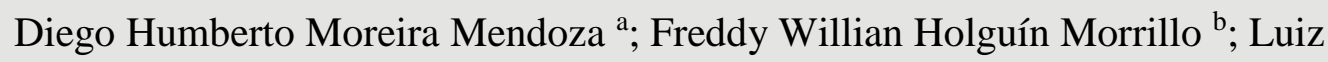
Carlos Flores Tacle ${ }^{c}$, Ronald Eugenio Cedeño Muñoz ${ }^{d}$;

Alex Ernesto Quimis Peña ${ }^{\mathrm{e}}$; Cristina Manuela Ramírez Matamoros ${ }^{\mathrm{f}}$

Prevención frente a síntomas que puede tener la mujer durante el climaterio

Prevention of symptoms that the woman may have during the climacteric

Revista Científica de Investigación actualización del mundo de las Ciencias. Vol. 3 núm., 1, enero, ISSN: 2588-0748, 2018, pp. 769-781

DOI: $\underline{10.26820 / \text { reciamuc/3.(1).enero.2019.769-781 }}$

URL: $\underline{\text { http://reciamuc.com/index.php/RECIAMUC/article/view/257 }}$

Código UNESCO: 3205 Medicina Interna

Tipo de Investigación: Artículo de Revisión

Editorial Saberes del Conocimiento

Recibido: 10/12/2018

Aceptado: 20/01/2019Publicado: 30/01/2019

Correspondencia: diwo345@gmail.com
a. Medico; diwo345@gmail.com; El Carmen, Ecuador
b. Médico Cirujano; freddyholguin@ @otmail.com; Ibarra, Ecuador
c. Doctor en Medicina; lucasfolclorico@yahoo.es; Ibarra, Ecuador
d. Médico Cirujano; recm1991@hotmail.com; Puerto Francisco de Orellana/El Coca, Ecuador
e. Médico; Kurt-1307@ hotmail.com; Pedernales, Ecuador
f. Médico; cristina_ramirez91@ @otmail.com; Guayaquil, Ecuador 


\section{Prevención frente a síntomas que puede tener la mujer durante el climaterio}

Vol. 3, núm. 1., (2019)

Diego Humberto Moreira Mendoza; Freddy Willian Holguín Morrillo; Luiz Carlos Flores Tacle; Ronald Eugenio Cedeño Muñoz; Alex Ernesto Quimis Peña; Cristina Manuela Ramírez Matamoros

\section{RESUMEN}

El climaterio es una etapa considerada en la vida de una mujer como un período de transición que abarca varios años, caracterizado por una baja significativa de la capacidad para producir estrógenos y agotamiento de los ovarios. Aunque climaterio y menopausia son términos que se han empleado como sinónimos, significan situaciones muy opuestas. La menopausia es un acontecimiento puntual en la vida de la mujer (el cese de la menstruación), mientras que el climaterio representa un período amplio que puede abarcar los 25-30 años entre la etapa reproductiva y la senectud. Los síntomas y signos del climaterio: sofocos, sudoración nocturna, sequedad, comezón, irritación o secreción vaginal, dolor, molestia durante el sexo, osteoporosis, problemas de control de la vejiga, infecciones de las vías urinarias, depresión, ansiedad, irritabilidad, cambios en el estado de ánimo y problemas para conciliar el sueño. Se recomienda conversar con su médico tratante respecto a los síntomas o malestares que padece con la finalidad de ubicar la alternativa más adecuada para la paciente, para los sofocos se recomienda la hipnosis, la meditación, en relación a los huesos finos, el ejercicio diario con su propio peso corporal como caminar durante 20 a 30 minutos por día o cualquier otra actividad física, tomar suplementos de calcio y/o vitamina D de ser requeridos. Para la resequedad vaginal puede usar humectante o lubricantes en la vagina. Las principales patologías que requieren atención especial en cualquier programa de salud de la mujer por el riesgo de aumento en el climaterio son: osteoporosis, enfermedades cardiovasculares: identificación de los factores de riesgo y su corrección, cáncer, la enseñanza y uso de la autoexploración de las mamas, mamografía periódica. La metodología usada es descriptiva, con un enfoque documental, es decir, revisar fuentes disponibles en la red, como google académico, con contenido oportuno y relevante desde el punto de vista científico que enriquezca el análisis del tema planteado en este artículo.

Palabras Claves: Prevención; Climaterio; Menopausia; Ejercicio; Alimentación Saludable; Terapia de Reemplazo Hormonal; Etapa; Calidad de Vida. 


\title{
Prevención frente a síntomas que puede tener la mujer durante el climaterio
}

Vol. 3, núm. 1., (2019)

Diego Humberto Moreira Mendoza; Freddy Willian Holguín Morrillo; Luiz Carlos Flores Tacle;

Ronald Eugenio Cedeño Muñoz; Alex Ernesto Quimis Peña; Cristina Manuela Ramírez

Matamoros

\begin{abstract}
The climacteric is a stage considered in the life of a woman as a period of transition spanning several years, characterized by a significant decrease in the ability to produce estrogen and exhaustion of the ovaries. Although climacteric and menopause are terms that have been used as synonyms, they mean very opposite situations. Menopause is a punctual event in a woman's life (the cessation of menstruation), while the climacteric represents a broad period that can cover 2530 years between the reproductive stage and old age. The symptoms and signs of the climacteric: hot flushes, night sweats, dryness, itching, irritation or vaginal discharge, pain, discomfort during sex, osteoporosis, bladder control problems, urinary tract infections, depression, anxiety, irritability, changes in the mood and trouble falling asleep. It is recommended to talk with your treating doctor about the symptoms or discomforts you suffer in order to locate the most appropriate alternative for the patient, for hot flashes hypnosis is recommended, meditation, in relation to fine bones, daily exercise with your own body weight such as walking for 20 to 30 minutes per day or any other physical activity, take calcium and / or vitamin D supplements if required. For vaginal dryness you can use moisturizer or lubricants in the vagina. The main pathologies that require special attention in any women's health program due to the risk of an increase in the climacteric are: osteoporosis, cardiovascular diseases: identification of risk factors and their correction, cancer, teaching and use of self-examination of the breasts, periodic mammography. The methodology used is descriptive, with a documentary approach, that is, to review sources available on the web, such as academic google, with timely and relevant content from a scientific point of view that enriches the analysis of the topic raised in this article.
\end{abstract}

Key Words: Prevention; Climacteric; Menopause; Exercise; Healthy Eating; Hormone Replacement Therapy; Stage; Quality of Life. 


\section{Prevención frente a síntomas que puede tener la mujer durante el climaterio}

Vol. 3, núm. 1., (2019)

Diego Humberto Moreira Mendoza; Freddy Willian Holguín Morrillo; Luiz Carlos Flores Tacle;

Ronald Eugenio Cedeño Muñoz; Alex Ernesto Quimis Peña; Cristina Manuela Ramírez Matamoros

\section{Introducción.}

Desde finales de la década de los sesenta ha existido un interés creciente en todo el mundo por la atención de la mujer en los períodos climatérico y postmenopáusico. Este interés nace, entre otros factores, del avance científico cada vez más grande acerca de las posibilidades de mejorar significativamente la calidad de vida en estos períodos, mediante acciones para la atención integral de la salud femenina, la supresión del síndrome climatérico, la reducción de la morbilidad y mortalidad vinculada con el incremento en los riesgos de osteoporosis y de enfermedad cardiovascular.

Durante muchos años el climaterio ha sido considerado, (desde una perspectiva social) como una desgracia para la mujer, percepción que sido alimentada por algunos profesionales de la salud, quienes han llegado a considerarlo una enfermedad, al catalogarlo como una endocrinopatía, basándose en que el hipoestronismo era el producto de un fracaso ovárico, sin tener en cuenta que este supuesto fracaso viene determinado genéticamente y depende de la dotación folicular que se establece durante la vida intrauterina.

Eliminar cualquier connotación de enfermedad o de patología ligado al acontecimiento fisiológico del climaterio y menopausia es uno de los aspectos más importantes a desarrollar desde cualquier ámbito de la salud y que más puede contribuir a eliminar una visión negativa de esta etapa a muchas mujeres. Este aspecto ligado a la educación sanitaria, podría solucionar muchos de los problemas que se asocian al climaterio, así como liberar a otras mujeres de algunos de los grandes mitos y tabúes que contribuyen a mantenerlas, todavía hoy día vigente.

En el presente artículo se abordará no solo sus síntomas y algunas medidas para sobre llevar las complicaciones que ocasionan estos cambios hormonales sino un conjunto de medidas que ayudarán a prevenir y contar con una mejor salud mientras se realiza el tránsito de esta etapa a la plenitud de la madurez de la mujer. 


\section{Prevención frente a síntomas que puede tener la mujer durante el climaterio}

Vol. 3, núm. 1., (2019)

Diego Humberto Moreira Mendoza; Freddy Willian Holguín Morrillo; Luiz Carlos Flores Tacle;

Ronald Eugenio Cedeño Muñoz; Alex Ernesto Quimis Peña; Cristina Manuela Ramírez

Matamoros

\section{Metodología.}

Esta investigación está dirigida al estudio "Prevención frente a síntomas que puede tener la mujer durante el climaterio". Para realizarlo se usó una metodología descriptiva, con un enfoque documental, es decir, revisar fuentes disponibles en la red, como google académico, con contenido oportuno y relevante desde el punto de vista científico para dar respuesta a lo tratado en el presente artículo y que sirvan de inspiración para realizar otros proyectos. Las mismas pueden ser estudiadas al final, en la bibliografía.

\section{Resultados.}

El climaterio es una etapa considerada en la vida de una mujer como un escalón entre el ciclo vital previo y posterior a la perdida de las funciones genitales. Es un período de transición que abarca varios años, caracterizado por una baja significativa de la capacidad para producir estrógenos y agotamiento de los ovarios.

Aunque climaterio y menopausia son términos que se han empleado como sinónimos, significan situaciones muy opuestas. La menopausia es un acontecimiento puntual en la vida de la mujer(el cese de la menstruación), mientras que el climaterio representa un período amplio que puede abarcar los 25-30 años entre la etapa reproductiva y la senectud(Dueñas, 1999).

La menopausia es un estado fisiológico de la mujer, parte del proceso natural de envejecimiento, caracterizado por el cese de la secreción hormonal ovárica, dando lugar a cambios que afectan el aparato urogenital, sistema cardiovascular y óseo. La Organización Mundial de la Salud (OMS) define la menopausia como el cese permanente de la menstruación, tras un periodo de doce meses consecutivos de amenorrea, sin otra causa aparente patológica ni psicológica. Se reserva el término de peri menopausia para el periodo de tiempo que precede a la última regla y los doce meses posteriores, y post menopausia para todos los años que siguen tras la última regla(Capote, 2011).

Para eliminar ideas que asocian el climaterio con un castigo o enfermedad, es necesario entenderlo como un período de transición absolutamente fisiológico, en el que tan sólo se producen algunos cambios adaptativos a una nueva situación biológica, consecuencia de la finalización de la 


\section{Prevención frente a síntomas que puede tener la mujer durante el climaterio}

Vol. 3, núm. 1., (2019)

Diego Humberto Moreira Mendoza; Freddy Willian Holguín Morrillo; Luiz Carlos Flores Tacle; Ronald Eugenio Cedeño Muñoz; Alex Ernesto Quimis Peña; Cristina Manuela Ramírez Matamoros

capacidad reproductiva, que se asocia con un cambio hormonal, al estar ligada la producción del estradiol con la existencia de folículos ováricos.

Con frecuencia se olvida que la mujer, anteriormente pasó por una situación similar, la adolescencia, período también de transición adaptativa entre la niñez y la madurez, necesario para adquirir la plena capacidad reproductiva y con la entrada en el juego endocrinológico de numerosos cambios hormonales mucho más complejos que los que se producen durante el climaterio. Para algunas personas, la mujer, a partir de la instauración de la menopausia, tendría menos valor social al no ser ya una reproductora, mientras que la adolescente incrementa su valor, al ser una joven reproductora. Se considera que en esta etapa tan temida por muchas mujeres, se alcanza la madurez, aparecen diversas manifestaciones físicas y emocionales(Velazco, 2000).

Síntomas y signos del climaterio (American Society of Clinical Oncology, 2019):

- Sofocos (mezcla súbita de calor y sudoración). Su rostro, pecho y otras áreas de su cuerpo pueden sentirse muy calientes. Esta sensación desaparece en unos minutos.

- Sudoración nocturna, se despierta durante la noche empapada de sudor.

- Sequedad, comezón, irritación o secreción vaginal.

- Dolor, molestia durante el sexo.

- Osteoporosis (afinamiento de los huesos, son más sensibles a romperse).

- Problemas de control de la vejiga, necesidad de orinar con frecuencia, dificultad para retener la orina o pérdidas.

- Infecciones de las vías urinarias.

- Depresión, ansiedad, irritabilidad y cambios en el estado de ánimo.

- Problemas para conciliar el sueño.

La inquietud hasta ahora es buscar abordar la atención de la mujer climatérica desde el primer nivel de salud. La respuesta es sencilla de dar, pero más compleja de realizar. Sencilla porque desde la atención primaria no hay más que asumir las necesidades de la mujer climatérica. En cualquier caso, y siguiendo el esquema de necesidades planteado anteriormente, el abordaje debería de ser el siguiente: 


\section{Prevención frente a síntomas que puede tener la mujer durante el climaterio}

Vol. 3, núm. 1., (2019)

Diego Humberto Moreira Mendoza; Freddy Willian Holguín Morrillo; Luiz Carlos Flores Tacle; Ronald Eugenio Cedeño Muñoz; Alex Ernesto Quimis Peña; Cristina Manuela Ramírez

Matamoros

Mejorar la calidad de vida de vida de la paciente no sólo cuando se presenten los síntomas del climaterio sino a lo largo de la vida, el acceso a la información es un elemento clave que disminuya la ansiedad e incertidumbre por esta etapa.

Hacer énfasis que no es una enfermedad sino un proceso fisiológico, los buenos hábitos presentes a lo largo de toda la vida incidirá en los síntomas y actitud de la mujer para asumir estos cambios. El alimentarse sanamente, la ingesta de suplementos de calcio, la práctica del ejercicio en forma regular y programado, mantener un peso adecuado a la edad y altura del paciente(Salvador, 2008).

La eliminación o disminución de hábitos negativos que repercuten en la salud de la paciente como el estrés, la tensión arterial elevada, la glicemia, la ingesta de café y bebidas alcohólicas. La terapia de sustitución de hormonas en las pacientes sintomáticas equilibrada, tomando en consideración el riesgo - beneficio de este tratamiento.

\section{Medidas para sobrellevar la fase de climaterio.}

Se recomienda conversar con su médico tratante respecto a los síntomas o malestares que padece con la finalidad de ubicar la alternativa más adecuada para la paciente, que considere sus gustos, posibilidades de acceso y económicas:

- Para los sofocos se recomienda hipnosis, terapia conductual o actividades de esparcimiento como la meditación.

- En relación a los huesos finos, el ejercicio diario con su propio peso corporal como caminar durante 20 a 30 minutos por día o cualquier otra actividad física que disfrute la paciente. Mantener un peso saludable. Tomar suplementos de calcio y/o vitamina D de ser requeridos. La densimetría ósea mide desgaste de huesos en lugares claves como las caderas y columna vertebral.

- Para la resequedad vaginal puede usar humectante o lubricantes en la vagina. Otros tratamientos recomendados: crema con estrógenos, anillos pequeños introducidos en la vagina, píldoras o capsulas que contienen estrógeno. 


\section{Prevención frente a síntomas que puede tener la mujer durante el climaterio}

Vol. 3, núm. 1., (2019)

Diego Humberto Moreira Mendoza; Freddy Willian Holguín Morrillo; Luiz Carlos Flores Tacle; Ronald Eugenio Cedeño Muñoz; Alex Ernesto Quimis Peña; Cristina Manuela Ramírez Matamoros

- Otra recomendación es la terapia de reemplazo hormonal si existe la necesidad de usarla la paciente. Si tiene útero, el tratamiento debe incluir progesterona junto con el estrógeno (esto impide el agrandamiento del útero).

Existe un grupo de estado clínico en el cual resulta poco conveniente la terapia hormonal debido a la existencia de mayores riesgos de salud que beneficios (Arriagada, 2005):

- Inicio en edad mayor o luego de largo tiempo de evolución de la menopausia (en mujeres asintomáticas).

- Estado pro trombótico o trombosis activa.

- Enfermedad coronaria.

- Accidente vascular encefálico.

- Historial personal del cáncer de mama.

- Sospecha de tumor estrógeno (hiperplasia endometrial, adenocarcinoma de endometrio o cuello uterino).

- Sangrado uterino de etiología no establecida.

- Insuficiencia hepática aguda.

\section{Prevención.}

Las principales patologías que requieren atención especial en cualquier programa de salud de la mujer por el riesgo de aumento en el climaterio son:

1. Osteoporosis: pueden emplearse baremos para la detección y cuantificación de los factores de riesgo para determinar el estado de la masa ósea, detección de la población de alto riesgo y seleccionar ésta para ser estudiada con técnicas más sofisticadas.

2. Enfermedades cardiovasculares: identificación de los factores de riesgo y su corrección, eliminando hábitos nocivos y tratando aquellos procesos concomitantes.

3. Cáncer: las neoplasias más frecuentes en la mujer climatérica son el cáncer de la mama, el de endometrio, ovario y, menos frecuente, el de cérvix uterino. Evaluando su frecuencia y pronóstico, es deseable establecer e incorporar a las mujeres climatéricas a programas de prevención del cáncer genital y mamario. 


\section{Prevención frente a síntomas que puede tener la mujer durante el climaterio}

Vol. 3, núm. 1., (2019)

Diego Humberto Moreira Mendoza; Freddy Willian Holguín Morrillo; Luiz Carlos Flores Tacle;

Ronald Eugenio Cedeño Muñoz; Alex Ernesto Quimis Peña; Cristina Manuela Ramírez

Matamoros

4. La enseñanza y uso de la autoexploración de las mamas, mamografía periódica son herramientas efectivas en el diagnostico precoz.

5. La existencia de alteraciones menstruales por exceso y metrorragias en las mujeres pre menopáusicas y la metrorragia en la mujer posmenopáusica, pueden sugerir carcinoma uterino, su detección debe implicar un adecuado estudio endocavitario (ecografía transvaginal, biopsia de endometrio, hieroscopia).

6. La ausencia de clínica precoz en el cáncer de ovario y su coexistencia con alteraciones digestivas y aumento del perímetro abdominal, son también datos de sospecha y motivo para un estudio más específico.

7. El cáncer de cérvix, que durante muchos años tuvo una frecuencia baja en la mujer posmenopáusica, está experimentando un importante incremento en esta etapa, por lo que recomienda mantenerse la práctica, en atención primaria, de citología periódica, al menos en mujeres con conductas de riesgo.

\section{Tratamiento.}

Estrógeno: la dosis de estrógeno debe ser la menor que alivie la sintomatología vasomotora, evite la pérdida ósea y prevenga la atrofia urogenital(Santana, 2007).Dosis de inicio:

\section{Tabla $\mathrm{N}^{\circ} 1$ Tratamiento hormonal para climaterio}

\begin{tabular}{|l|l|}
\hline Dosis & Tratamiento \\
\hline $\mathbf{0 . 5 - 1 . 0}(\mathbf{m g})$ & Estradiol oral \\
\hline $\mathbf{0 . 3 - 0 . 6 2 5}(\mathbf{m g})$ & Estrógeno conjugado \\
\hline $\mathbf{2 5 - 5 0}$ ug & Estradiol trandérmico \\
\hline $\mathbf{0 . 5 - 1 . 0}(\mathbf{m g})$ & Estradiol gel \\
\hline
\end{tabular}

Fuente: (Arriagada, 2005)

La dosis puede ser ajustada aproximadamente a los 3 meses dependiendo de la respuesta sintomática. 


\section{Prevención frente a síntomas que puede tener la mujer durante el climaterio}

Vol. 3, núm. 1., (2019)

Diego Humberto Moreira Mendoza; Freddy Willian Holguín Morrillo; Luiz Carlos Flores Tacle;

Ronald Eugenio Cedeño Muñoz; Alex Ernesto Quimis Peña; Cristina Manuela Ramírez

Matamoros

\section{Duración.}

La duración depende de la indicación del tratamiento. El tipo, la dosis y la vía de administración deben ser reevaluados anualmente. La mantención del tratamiento para el alivio de la sintomatología puede ser evaluada con la suspensión temporal de la terapia (o disminución de la dosis) después de 2 ó 3 años de uso.Para prevención y tratamiento de osteoporosis la TH es una buena alternativa. En el tratamiento de osteoporosis a largo plazo deben considerarse alternativas terapéuticas no hormonales. El uso de estrógenos de acción local por vía vaginal puede indicarse a cualquier edad y utilizarse por períodos prolongados de tiempo para alivio de la sintomatología urogenital (Abellán, 2002).

\section{Progesterona.}

- En mujeres con útero se debe usar una progestina para proteger el endometrio.

- No se requiere el uso de progestina en pacientes sin útero.

- En esquemas secuenciales la progestina debe ser usada en dosis adecuada por 10-14 días al mes.

- La evidencia médica actual aún no avala el uso de progestinas en ciclos largos cada 3 meses.

- Con dosis baja de sustitución estrogénica se puede utilizar dosis menores de progestina.

- El uso de progestinas por vía vaginal parece una interesante alternativa, pero su seguridad aún no se encuentra suficientemente respaldada.

- Existe información creciente que las progestinas comúnmente usadas en TH están involucradas en el riesgo de cáncer de mama y afectan negativamente los factores de riesgo cardiovascular.

\section{Aplicación:}

La vía oral es la de mayor experiencia. Tiene como desventaja el efecto de primer paso hepático que puede provocar un aumento de factores de coagulación, triglicéridos, angiotensinógeno y 


\section{Prevención frente a síntomas que puede tener la mujer durante el climaterio}

Vol. 3, núm. 1., (2019)

Diego Humberto Moreira Mendoza; Freddy Willian Holguín Morrillo; Luiz Carlos Flores Tacle; Ronald Eugenio Cedeño Muñoz; Alex Ernesto Quimis Peña; Cristina Manuela Ramírez

Matamoros

proteína $\mathrm{C}$ reactiva. La vía transdérmica es de elección en pacientes con hipertrigliceridemia, enfermedad hepática y migraña. No aumenta la PCR y no aumentaría los factores de coagulación, puede ser ventajosa en hipertensas. Tiene como desventaja la posibilidad de irritación dérmica. La vía vaginal es adecuada y segura para mujeres con sintomatología urogenital y sin sintomatología climatérica sistémica.

\section{Tibolona.}

Es un esteroide sintético clasificado como STEAR (regulador tisular selectivo de la actividad estrogénica), siendo una pro-droga con actividad progestagénica, androgénica y estrogénica. Alivia los síntomas vasomotores de la menopausia, protege los tejidos urogenitales, tiene efectos beneficiosos sobre el estado de ánimo y la libido, conserva la masa ósea, y no aumenta la densidad mamaria radiológica en la mayoría de las usuarias. Su efecto sobre el riesgo de cáncer mamario no se encuentra aclarado. Su efecto sobre la prevención de fracturas aún no se ha establecido, pero está siendo evaluada.

\section{SERMs (Raloxifeno).}

Sugerido para la prevención y el tratamiento de la osteoporosis; disminuye el riesgo de fracturas vertebrales en mujeres postmenopáusicas osteoporótica. Sin acción sobre la sintomatología vasomotora ni sobre el tejido urogenital. Existen estudios en desarrollo sobre su efecto sobre el sistema cardiovascular y en quimio prevención de cáncer de mama. En el estudio MORE en mujeres osteoporótica, demostró reducción del riesgo cardiovascular en mujeres con factores de riesgo cardiovascular y prevención de cáncer de mama.

\section{Fitoestrógenos.}

No son considerados medicamentos, por lo que no se les exige estudios de eficacia y seguridad. La mayoría de los estudios clínicos publicados no demuestra acción significativa sobre los síntomas vasomotores y sobre el hueso. Tampoco se encuentra suficientemente avalada su seguridad clínica. 


\section{Prevención frente a síntomas que puede tener la mujer durante el climaterio}

Vol. 3, núm. 1., (2019)

Diego Humberto Moreira Mendoza; Freddy Willian Holguín Morrillo; Luiz Carlos Flores Tacle; Ronald Eugenio Cedeño Muñoz; Alex Ernesto Quimis Peña; Cristina Manuela Ramírez Matamoros

\section{Conclusiones.}

Durante muchos años el climaterio ha sido considerado como una desgracia para la mujer, percepción que sido alimentada por algunos profesionales de la salud hace muchos años atrás, quienes han llegado a considerarlo una enfermedad, al catalogarlo como una endocrinopatía, basándose en que el hipoestronismo era el producto de un fracaso ovárico, sin tener en cuenta que este supuesto fracaso viene determinado genéticamente y depende de la dotación folicular que se establece durante la vida intrauterina.

Eliminar cualquier connotación de enfermedad o de patología ligado al acontecimiento fisiológico del climaterio y menopausia es uno de los aspectos más importantes a desarrollar desde cualquier ámbito de la salud y que más puede contribuir a eliminar una visión negativa de esta etapa a muchas mujeres. La educación sanitaria, podría solucionar muchos de los problemas que se asocian al climaterio, así como liberar a otras mujeres de algunos de los grandes mitos y tabúes que contribuyen a mantenerlas, todavía hoy día vigente.

Mejorar la calidad de vida de vida de la paciente no sólo cuando se presenten los síntomas del climaterio sino a lo largo de la vida, el acceso a la información es un elemento clave que disminuya la ansiedad e incertidumbre por esta etapa. Hacer énfasis que no es una enfermedad sino un proceso fisiológico, los buenos hábitos presentes a lo largo de toda la vida incidirá en los síntomas y actitud de la mujer para asumir estos cambios. El alimentarse sanamente, la ingesta de suplementos de calcio, la práctica del ejercicio en forma regular y programado, mantener un peso adecuado a la edad y altura del paciente.

La eliminación o disminución de hábitos negativos que repercuten en la salud de la paciente como el estrés, la tensión arterial elevada, la glicemia, la ingesta de café y bebidas alcohólicas. La terapia de sustitución de hormonas en las pacientes sintomáticas equilibrada, tomando en consideración el riesgo - beneficio de este tratamiento. 


\section{Prevención frente a síntomas que puede tener la mujer durante el climaterio}

Vol. 3, núm. 1., (2019)

Diego Humberto Moreira Mendoza; Freddy Willian Holguín Morrillo; Luiz Carlos Flores Tacle; Ronald Eugenio Cedeño Muñoz; Alex Ernesto Quimis Peña; Cristina Manuela Ramírez

Matamoros

\section{Bibliografía.}

Abellán, J. (Mayo de 2002). Climaterio y riesgo cardiovascular. Revista de Nefrología, 105-111. www.revistenefrología.com.

American Society of Clinical Oncology. (Enero de 2019). Síntomas de la menospausia en las mujeres. Obtenido de Cancer.Net: www.cancer.net

Arriagada, M. (2005). Recomendaciones de tratamiento durante la menospausia. Revista Chilena de Ginecología y Obstetricia. 70(5). 340-345.

Capote, M. (2011). Climaterio y Menospausia. Revista Cubana de Medicina General Integral, Volumen $27 N^{\circ} 4$, www.scielo.com.cu.

Dueñas, J. (1999). Atención a la menospausia desde el primer nivel de salud. Estadísticas Volumen 23, $N^{\circ} 6,323-389$ www.elsevier.com.

Salvador, J. (Abril de 2008). Climaterio y menopausia. Obtenido de Spog: www.spog,org.pe

Santana, F. (2007). Efecto de la menopausia sobre la prolactina de las mujeres. Revista Cubana de Endocrinología. 18(2)

Velazco, V. (2000). Experiencias y conocimientos sobre climaterio y menospausia en mujeres de Ciudad de Mejico. Gaceta Médica de Méjico. 136(6). 555-564. 